\title{
Sentence Simplification from Non-Parallel Corpus with Adversarial Learning
}

\author{
Takashi Kawashima \\ t_kawashima@cs.meiji.ac.jp \\ Dept. of Computer Science \\ Meiji University \\ Japan
}

\author{
Tomohiro Takagi \\ takagi@cs.meiji.ac.jp \\ Dept. of Computer Science \\ Meiji University \\ Japan
}

\begin{abstract}
In this study, we propose sentence simplification from a non-parallel corpus with adversarial learning. In recent years, sentence simplification based on a statistical machine translation framework and neural networks have been actively studied. However, most methods require a large parallel corpus, which is expensive to build. In this paper, our purpose is sentence simplification with a nonparallel corpus in open data en-Wikipedia and Simple-Wikipedia articles. We use a style transfer framework with adversarial learning for learning by non-parallel corpus and adapted a prior work [by Barzilay et al.] to sentence simplification as a base framework. Furthermore, from the perspective of improving retention of sentence meaning, we add pretraining reconstruction loss and cycle consistency loss to the base framework. We also improve the sentence quality output from the proposed model as a result of the expansion
\end{abstract}

\section{CCS CONCEPTS}

- Computing methodologies $\rightarrow$ Natural language generation.

\section{KEYWORDS}

sentence simplification, adversarial learning, non-parallel corpus

\section{ACM Reference Format:}

Takashi Kawashima and Tomohiro Takagi. 2019. Sentence Simplification from Non-Parallel Corpus with Adversarial Learning. In IEEE/WIC/ACM International Conference on Web Intelligence (WI '19), October 14-17, 2019, Thessaloniki, Greece. ACM, New York, NY, USA, 8 pages. https://doi.org/10. $1145 / 3350546.3352499$

\section{INTRODUCTION}

The main purpose of sentence simplification is to reduce the linguistic complexity of the sentence while preserving the information and meaning of the original sentence. Sentence simplification supports many readers who are learning a language (including children) and is essential in NLP because sentence simplification is sometimes used for improving the performance of parsers [5]

Permission to make digital or hard copies of all or part of this work for personal or classroom use is granted without fee provided that copies are not made or distributed for profit or commercial advantage and that copies bear this notice and the full citation on the first page. Copyrights for components of this work owned by others than ACM must be honored. Abstracting with credit is permitted. To copy otherwise, or republish, to post on servers or to redistribute to lists, requires prior specific permission and/or a fee. Request permissions from permissions@acm.org.

WI '19, October 14-17, 2019, Thessaloniki, Greece

(C) 2019 Association for Computing Machinery.

ACM ISBN 978-1-4503-6934-3/19/10 . \$ \$15.00

https://doi.org/10.1145/3350546.3352499 and summary task [17]. There are two linguistic aspects of operation for the sentence simplification: (a) the lexical aspect, which is to replace complex words in a sentence with simple words or idioms [2][3][4][10][13][15][40], and (b) the syntactic aspect, which is to compress complex sentence structures and delete original elements [4][8][26]. Earlier work focused on these individual aspects of the simplification problem. From the perspective of sentence construction, sentence simplification can be considered a form of text-transformation that has three main operations: (a) splitting [22], (b) deletion/compression [8][28], and (c) paraphrasing [33].

There are two main approaches to sentence simplification. The first approach is a method that generates a simple sentence from an input sentence by a statistical machine translation framework that interprets monolingual machine translation [22][36][34][35][46]. The second approach is a method using a neural network [29][32][43]. In other NLP tasks (e.g., QA task, word embedding), the neural network method has had excellent results and is being actively researched. However, most of these techniques require a large parallel corpus for the training model. It is difficult to obtain sufficient data volume about a simplification parallel corpus because the construction of the monolingual parallel corpus necessary for training a sentence simplification model is expensive, unlike machine translation to a different language. Moreover, the machine translation task [1] has an unsupervised model with data-driven generation.

To avoid the expense of creating a large parallel corpus about sentence simplification, we simplify sentences from a non-parallel corpus with adversarial learning in the framework of unsupervised style transfer in NLP. The style transfer task is being actively researched in the fields of NLP and image processing. In particular, style transfer in NLP has sentiment modification, word substitution decipherment, and so on. The style transfer task is frequently taken with adversarial learning not requiring a parallel corpus because it is difficult to obtain a large parallel corpus. Generative Adversarial Nets (GAN) learn by means of an adversarial generator and discriminator. The generator tries to generate real data, and the discriminator distinguishes between the generator's output and real data. GAN developed rapidly from the proposal in [11] in the field of image generation. We adapt [30] with adversarial learning to sentence simplification from a non-parallel corpus. Furthermore, to improve the retention of sentence meaning, we add pretraining reconstruction loss and cycle consistency loss to the base model [30]. The pretraining teaches the generator to output the same sentence as the input sentence. The cycle consistency loss compares the difference of input sentence and output sentence by transferring twice (i.e., complex $\rightarrow$ simple $\rightarrow$ complex). We compare [30] as a Base 
model, Base+Pretrain model, and Base+Pretrain+Cycle model. We use FRE difference, BLEU, and SARI for the quantitative evaluation. The results show that our proposed model (Base+Pretrain+Cycle) had a better performance for BLEU and SARI than the other models, and the comparison in the output sentence was a more effective simplification than the other models.

\section{RELATED WORK}

\subsection{Sentence Simplification}

Sentence simplification has often been discussed from the psychological and linguistic point of view. Recently it has become a mainstream research topic in computational linguistics since [26] proposed a model to automatically extract a simplification rule from an annotated corpus. [42] proposed a sentence simplification method that uses a morphological analyzer and part-of-speech tagger from both lexical and syntactical aspects. However, these methods cannot paraphrase phrase-base because most of them only perform operations such as word substitution and sentence separation or deletion. As mentioned above, there are two main approaches to sentence simplification. The first is a method that generates simple sentences from an input sentence by a statistical machine translation framework that interprets monolingual machine translation. The second approach is a method that uses a neural network. It is now possible to create paraphrase-based sentence simplification since data-driven sentence simplification has appeared.

In the case of statistical methods, [46] proposed a tree-based model inspired by statistical machine translation. [34] formulated simplification in the framework of quasi-synchronous grammar and used integer linear programming to score sentence simplification rules. [35] proposed a PBMT-R with two stages. PBMT-R trains a phrase-based machine translation model from normal-simple aligned sentence pairs and reranks the generated sentences by comparing them with a normal sentence by dissimilarity. [22] also proposed a model with two stages: deep semantic analysis and machine translation. [36] adapted syntax-based machine translation with a large parallel corpus to sentence simplification. [23] proposed an unsupervised model to replace complex words with simple words.

In the case of neural network methods, [43] enabled sentence simplification by deep reinforcement learning in which simplicity, relevance, and fluency are considered in the reward. [32] adapted a sequence-to-sequence model with a Neural Semantic Encoder [31] for the purpose of sentence simplification. [29] is a simple sequenceto-sequence model that can be efficiently used for in-domain and cross-domain sentence simplification.

\subsection{Generative Adversarial Nets}

In the General Adversarial Nets (GAN) proposed by [11], the discriminator learns to distinguish between the generator's output and real data and the generator learns to mislead the discriminator by generating data that seems real. This approach has many applications in the field of image processing. [27] generated images from detailed textual descriptions. [20] utilized weight-sharing between networks for learning cross-domain representation and enabled style transfer of images. [45] proposed CycleGAN, which enables image-to-image transfer from an unsupervised framework by removing pair restriction of train data.

In contrast to GAN in NLP, [41] proposed a model that extends GAN to sequential data. In recent years, GAN in NLP is being actively studied. Regarding text generation, [44] proposed TextGAN using RNN that performs optimization in a high-dimensional latent space. [7] proposed MaskGAN masking encode so that it can be recovered from unknown input at decoding. In the field of dialogue, [19] evaluated whether the generated utterance can be distinguished from human beings (like Turing testing) and used reinforcement learning to give rewards for each generated word. [37] proposed a model not using reinforcement learning but rather an approximate layer. This model doesn't need sampling. In the field of machine translation, [38] is a conventional model that applies GAN to neural machine translation and a discriminator to determine whether it is a system translation or manual. In [6], a discriminator identifies the presence or absence of noise and improves the robustness of neural machine translation.

\subsection{Style Transfer in Natural Language}

The style transfer task in the parallel corpus is often regarded as a monolingual machine translation task. At this time, many sequence-to-sequence models have been adapted and achieved good results. For example, [14] proposed a sequence-to-sequence model to convert modern standard English to Shakespearean English. [25] used the framework of back translation for style transfer.

On the other hand, because of the lack of a parallel corpus, research on the style transfer model in a non-parallel corpus that does not require a parallel corpus is also popular in recent years. [12] used a variational autoencoder that has a discriminator to generate sentences with controllable attributes. [30] used an encoder that maps the original sentence to style-independent variables and a decoder that converts it to the specified style. [39] applied the language model of the target domain in a discriminator to convert more fluency. [18] proposed deleting words related with the source style, retrieving similar sentences, and replacing them with similar phrases related with the target style.

\section{MODEL DESCRIPTION}

In this paper, based on the method of [30] adapted to style transfer, we propose sentence simplification with GAN. We define two corpora of different styles as $X_{0}=\left\{x_{1}^{0}, \ldots, x_{m}^{0}\right\}, X_{1}=\left\{x_{1}^{1}, \ldots, x_{n}^{1}\right\}$. Neither corpus has to be parallel and has style values $s_{0}, s_{1}$ (e.g., complex and simple). K-th sentence in corpus $X_{i}$ with style $s_{i}$ writes $x_{k}^{i}$ to generalize. In 3.1, we formulate the base model. In 3.2 and 3.3, we formulate the extension by introducing the proposed model pretraining and cycle consistency loss to the base model.

\subsection{Base Model}

3.1.1 Generator. The generator includes two mechanisms: encoder $E$ and decoder $G$. We denote latent variable output $z_{k}^{i}$ regarding $E$ as $E\left(x_{k}^{i}, s_{i}\right)=z_{k}^{i}$ when sentence $x_{k}^{i}$ and style $s_{i}$ are input. We denote the sequence of latent variable output $H_{k}^{i \rightarrow j}$ regarding $G$ as $G\left(\tilde{h}_{i, k}^{0}\right)=H_{k}^{i \rightarrow j}$ when latent variable $z_{k}^{i}$ and style $s_{j}$ are input $\left(\tilde{h}_{i, k}^{0}=\left[z_{k}^{i}, s_{j}\right]\right)$ and $H_{k}^{i \rightarrow j}=\left\{\tilde{h}_{i, k}^{1}, \ldots, \tilde{h}_{i, k}^{T}\right\}$. We add a tilde to the $h$ 


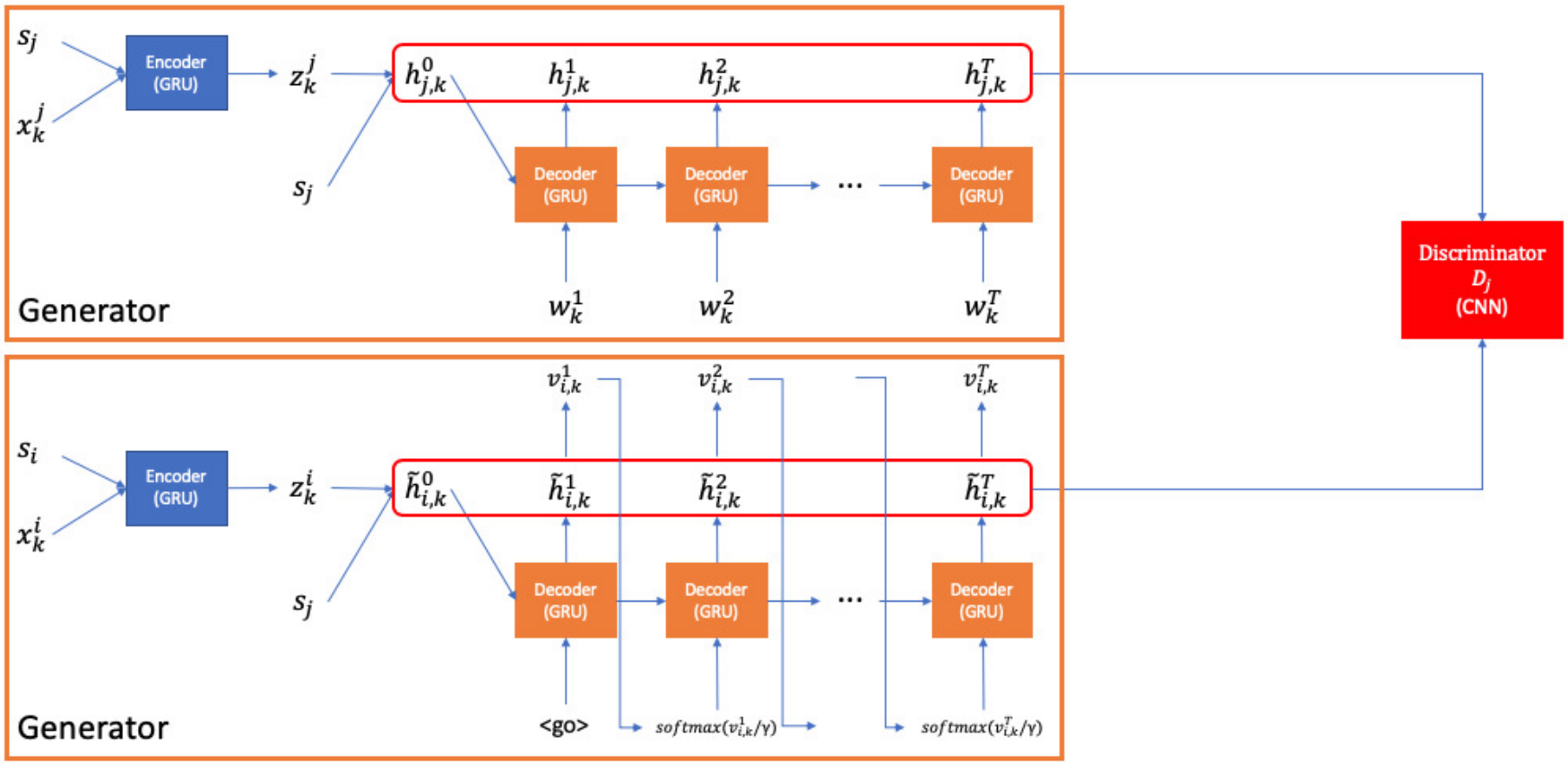

Figure 1: Discriminator overview about $D_{j}$.

if the style has changed. $T$ is the number of $G$ 's latent variant output. $E$ and $G$ use GRU. In contrast, we denote a teacher-forced process (upper part of Fig. 1) when we write $G\left(h_{i, k}^{0}, x_{k}^{i}\right)\left(\operatorname{add} x_{k}^{i}\right.$ to $\left.G\right)$. Let $\hat{x}_{k}^{i \rightarrow j}$ be generated with $H_{k}^{i \rightarrow j}$ and logit vector $v_{i, k}^{t}$ be calculated with $\tilde{h}_{i, k}^{t}$ at $t$ step. Decoder's input is $\operatorname{softmax}\left(v_{i, k}^{t} / \gamma\right)$ (lower part of Fig. 1) and $\gamma \in(0,1)$ is the temperature parameter. $X_{i \rightarrow j}$ is the sentence set that includes input sentence $x_{k}^{i}$ (style $s_{i}$ ) converted into sentence $\hat{x}_{k}^{i \rightarrow j}$ (style $s_{i} \rightarrow s_{j}$ ). If $j=i$, let $H_{k}^{i \rightarrow i}=\left\{h_{i, k}^{1}, \ldots, h_{i, k}^{T}\right\}$ be the decoder's latent variant and sequence of output and $\hat{x}_{k}^{i \rightarrow i}$ be the output sentence. Also, the generator output sentence is converted from the style of the input sentence to the target style in order to mislead the discriminator.

3.1.2 Discriminator. Next, we explain the discriminator for adversarial learning, as shown in Fig. 1. This discriminator uses CNN, the same as [30]. The discriminator includes $D_{i}, D_{j}$ and identifies whether the style of the input sentence has been converted or not. In the following, we explain $D_{j}$, but the same applies to $D_{i}$. The ideal discriminator $D_{j}$ 's output is probability value. The probability is close to 1 if the input is what style of the sentence doesn't change $\left(s_{j} \rightarrow s_{j}\right)$ and close to 0 if it does change $\left(s_{i} \rightarrow s_{j}\right)$. We denote $D_{j}\left(\tilde{h}_{i, k}^{0}, H_{k}^{i \rightarrow j}\right)=p_{D_{j}}\left(s_{j} \mid \tilde{h}_{i, k}^{0}, H_{k}^{i \rightarrow j}\right)$ if $D_{j}$ 's input is $\tilde{h}_{i, k}^{0}, H_{k}^{i \rightarrow j}$ and $D_{j}\left(h_{j, k}^{0}, H_{k}^{j \rightarrow j}\right)=p_{D_{j}}\left(s_{j} \mid h_{j, k}^{0}, H_{k}^{j \rightarrow j}\right)$ in the case of $s_{j} \rightarrow s_{j}$.

3.1.3 Loss Function. In the following, we explain reconstruction loss and adversarial loss. Reconstruction loss $\mathcal{L}_{\text {rec }}$ is the difference between sentence input and output (like autoencoder) and is calculated using input sentence $x_{k}^{i}, x_{k}^{j}$ and output $\hat{x}_{k}^{i \rightarrow i}, \hat{x}_{k}^{j \rightarrow j}$ :

$$
\begin{array}{r}
\mathcal{L}_{r e c}=\mathbb{E}_{x_{k}^{i} \sim X_{i}}\left[-\log p_{G}\left(x_{k}^{i} \mid E\left(x_{k}^{i}, s_{i}\right), s_{i}\right)\right]+ \\
\mathbb{E}_{x_{k}^{j} \sim X_{j}}\left[-\log p_{G}\left(x_{k}^{j} \mid E\left(x_{k}^{j}, s_{j}\right), s_{j}\right)\right]
\end{array}
$$

Adversarial loss $\mathcal{L}_{\text {adv }}$ is low value if the discriminator's performance is good and high value if the generator's performance is good. Adversarial loss $\mathcal{L}_{a d v_{i}}$ regarding $s_{i}$ is calculated as

$$
\begin{aligned}
\mathcal{L}_{a d v_{i}} & =\mathbb{E}_{x_{k}^{i} \sim X_{i}}\left[-\log D_{i}\left(\left[E\left(x_{k}^{i}, s_{i}\right), s_{i}\right], G\left(h_{i, k}^{0}, x_{i}\right)\right)\right]+ \\
& \mathbb{E}_{x_{k}^{j} \sim X_{j}}\left[-\log \left(1-D_{i}\left(\left[E\left(x_{k}^{j}, s_{j}\right), s_{i}\right], G\left(\tilde{h}_{j, k}^{0}\right)\right)\right)\right],
\end{aligned}
$$

and adversarial loss $\mathcal{L}_{a d v_{j}}$ regarding $s_{j}$ is calculated as

$$
\begin{aligned}
\mathcal{L}_{a d v_{j}} & =\mathbb{E}_{x_{k}^{j} \sim X_{j}}\left[-\log D_{j}\left(\left[E\left(x_{k}^{j}, s_{j}\right), s_{j}\right], G\left(h_{j, k}^{0}, x_{j}\right)\right)\right]+ \\
& \mathbb{E}_{x_{k}^{i} \sim X_{i}}\left[-\log \left(1-D_{j}\left(\left[E\left(x_{k}^{i}, s_{i}\right), s_{j}\right], G\left(\tilde{h}_{i, k}^{0}\right)\right)\right)\right],
\end{aligned}
$$

During training, $E$, $G$, and $D$ aim to minimize or maximize losses as follows:

$$
\mathcal{L}\left(\theta_{E}, \theta_{G}, \theta_{D}\right)=\min _{E, G} \max _{D} \mathcal{L}_{r e c}-\lambda\left(\mathcal{L}_{a d v_{i}}+\mathcal{L}_{a d v_{j}}\right),
$$

where $\theta_{E}, \theta_{G}, \theta_{D}$ are parameters of $E, G, D$, respectively.

\subsection{Model Pretrain}

As one element of the proposed model, we expand the base model to learn how to minimize $\mathcal{L}_{\text {rec }}$ before adversarial learning. To 


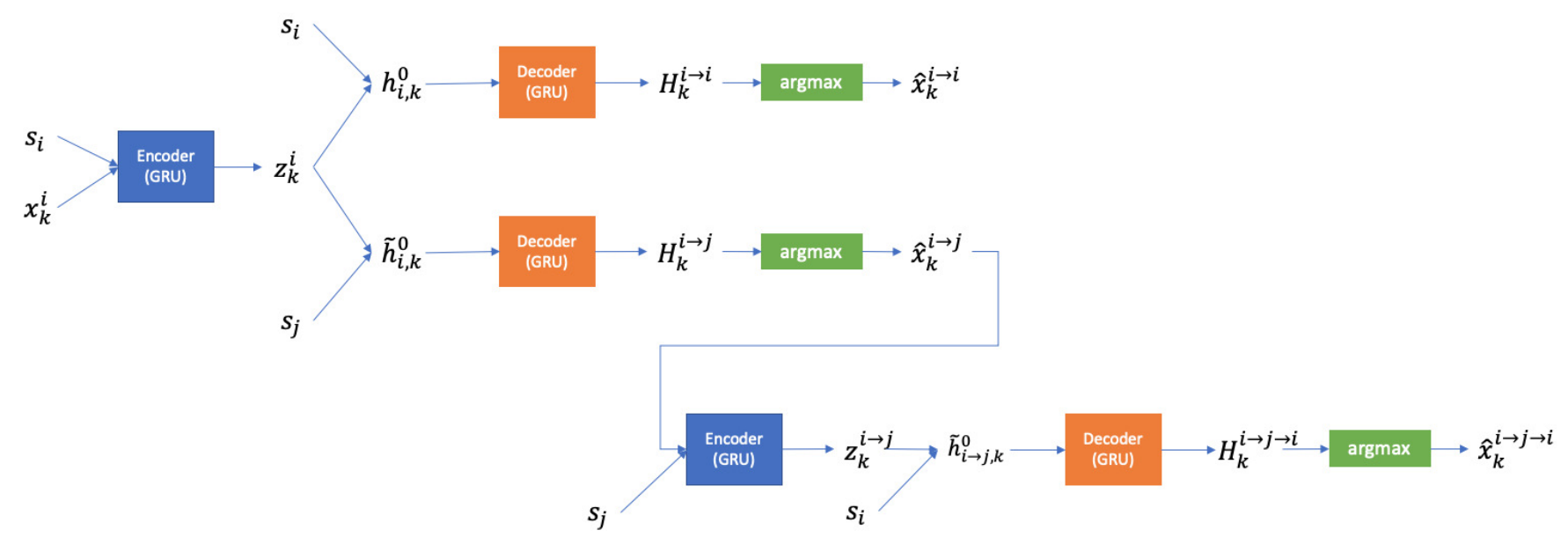

Figure 2: Generator overview.

minimize $\mathcal{L}_{\text {rec }}$, decrease the difference between input and output (as explained in 3.1.3). Therefore, this process improves retention of sentence content.

\subsection{Cycle Consistency Model}

3.1 cannot calculate the loss regarding the sentence transferred style $\left(X_{i \rightarrow j}, X_{j \rightarrow i}\right)$. We introduce cycle consistency loss to the model of 3.2 to resolve this problem. This process improves retention of sentence content by imposing the constraints on the generated sentences. An overview of the generator used in the proposed model is shown in Fig. 2. We denote the latent variable output $z_{k}^{i \rightarrow j}$ regarding $E$ as $E\left(\hat{x}_{k}^{i \rightarrow j}, s_{j}\right)=z_{k}^{i \rightarrow j}$ when sentence $\hat{x}_{k}^{i \rightarrow j}$ and style $s_{j}$ are input. We denote the sequence of latent variable output $H_{k}^{i \rightarrow j \rightarrow i}$ regarding $G$ as $G\left(\tilde{h}_{i \rightarrow j, k}^{0}\right)=H_{k}^{i \rightarrow j \rightarrow i}$ when latent variable $z_{k}^{i \rightarrow j}$ and style $s_{i}$ are input $\left(\tilde{h}_{i \rightarrow j, k}^{0}=\left[z_{k}^{i \rightarrow j}, s_{i}\right]\right)$. Let $\hat{x}_{k}^{i \rightarrow j \rightarrow i}$ be the sentence generated by $H_{k}^{i \rightarrow j \rightarrow i}$. Cycle consistency loss $\mathcal{L}_{c y c}$ is calculated by

$$
\begin{array}{r}
\mathcal{L}_{c y c}=\mathbb{E}_{x_{k}^{i} \sim X_{i}}\left[-\log p_{G}\left(x_{k}^{i} \mid E\left(\hat{x}_{k}^{i \rightarrow j}, s_{j}\right), s_{i}\right)\right]+ \\
\mathbb{E}_{x_{k}^{j} \sim X_{j}}\left[-\log p_{G}\left(x_{k}^{j} \mid E\left(\hat{x}_{k}^{j \rightarrow i}, s_{i}\right), s_{j}\right)\right]
\end{array}
$$

Adversarial loss $\mathcal{L}_{c y c \_a d v_{i}}$ regarding $\hat{x}^{i \rightarrow j \rightarrow i}$ is calculated by

$$
\mathcal{L}_{c y c \_a d v_{i}}=\mathbb{E}_{\hat{x}_{k}^{i \rightarrow j} \sim X_{i \rightarrow j}}\left[-\log \left(1-D_{i}\left(\left[E\left(\hat{x}_{k}^{i \rightarrow j}, s_{j}\right), s_{i}\right], G\left(\tilde{h}_{i \rightarrow j, k}^{0}\right)\right)\right)\right],
$$

and adversarial loss $\mathcal{L}_{c y c \_a d v_{j}}$ regarding $\hat{x}^{j \rightarrow i \rightarrow j}$ is caculated by

$$
\mathcal{L}_{c y c_{-} a d v_{j}}=\mathbb{E}_{\hat{x}_{k}^{j \rightarrow i} \sim X_{j \rightarrow i}}\left[-\log \left(1-D_{j}\left(\left[E\left(\hat{x}_{k}^{j \rightarrow i}, s_{i}\right), s_{j}\right], G\left(\tilde{h}_{j \rightarrow i, k}^{0}\right)\right)\right)\right]
$$

Therefore, our proposed model (Base+Pretrain + Cycle model) is optimized by

$$
\begin{array}{r}
\mathcal{L}\left(\theta_{E}, \theta_{G}, \theta_{D}\right)=\min _{E, G} \max _{D} \mathcal{L}_{r e c}+\mathcal{L}_{c y c} \\
-\lambda\left(\mathcal{L}_{a d v_{i}}+\mathcal{L}_{a d v_{j}}+\mathcal{L}_{c y c_{-} a d v_{i}}+\mathcal{L}_{c y c_{-} a d v_{j}}\right)
\end{array}
$$

\section{TRAINING ALGORITHM}

The algorithm of our proposed model (Base+Pretrain+Cycle model) is Algorithm 1.

Algorithm 1 Unsupervised simplification algorithm by cycle consistency model. The hyper-parameters are set as $\lambda=0.05, \gamma=0.001$ and learning rate is 0.0001 for all experiments.

Input: Two corpora of different styles $X_{0}, X_{1}$ (e.g., complex and simple). Initialize $\theta_{E}, \theta_{G}, \theta_{D_{0}}, \theta_{D_{1}}$

Pretrain:

Train embedding model using $X_{0}, X_{1}$

Train $E, G$ using $\mathcal{L}_{r e c}$

Adversarial Learning:

repeat

Train $D_{0}$ using $\mathcal{L}_{a d v_{0}}, \mathcal{L}_{c y c_{-} a d v_{0}}$

Train $D_{1}$ using $\mathcal{L}_{a d v_{1}}, \mathcal{L}_{c y c_{-} a d v_{1}}$

Train $E, G$ using $\mathcal{L}_{r e c}, \mathcal{L}_{c y c}$

until specified number of epochs are finished or convergence

Let input sentence $x_{k}$ be word sequence $x_{k}=\left\{w_{k}^{1}, \ldots, w_{k}^{T}\right\}\left(w_{k}^{t}\right.$ is word at $t$ step). We introduce word2vec [21] to $w_{k}^{t}$ for embedding pretrain. Word2vec can be considered the similarity of the meaning of the word. Therefore, it is effective for style transfer that maintains the meaning of the sentence. The data for embedding pretrain is a combined corpus of $s_{i}$ and $s_{j}$.

$\mathcal{L}_{\text {rec }}, \mathcal{L}_{\text {cyc }}$ are the difference between input and output. This input to $E$ is a random word sequence while the model is training. This process, called denoising, changes the word sequence to $E$ and compares true input and output while calculating loss. Therefore, denoising improves the grammaticality. 
$\lambda$ in Eqs. (4) and (8) is a hyperparameter to control adversarial loss. $\lambda$ of high value emphasizes adversarial loss while $\lambda$ of low value emphasizes $\mathcal{L}_{r e c}, \mathcal{L}_{c y c}$. [30]'s default value is $\lambda=1$, but our value is $\lambda=0.05$, as we prioritize maintaining sentence meaning.

\section{EXPERIMENT}

\subsection{Dataset}

5.1.1 Train Data. In order to train the model for sentence simplification, we made a non-parallel corpus of simple-Wikipedia and en-Wikipedia articles. The following conditions were added to simplify the problem when extracting sentences:

(1) Exclude sentences that contain symbols other than the period (.) and comma (,).

(2) The number of words in the sentence is more than four or less than 15.

When processing the corpus, processing was performed under the following conditions:

(1) Convert numbers to _num_.

(2) Unify all words into lower case.

(3) If a word occurs in both corpora five times or less, change to $<$ unk $>$.

We used Flesch Readability Ease (FRE) [9] to separate complex sentences and simple sentences more clearly. FRE is a metric in which the higher the numerical value, the easier it is to read a sentence. The sentences in the en-Wikipedia corpus, which have an FRE of 25 or less, are the complex corpus, and the sentences in the simple-Wikipedia corpus, which have an FRE of 75 or more, are the simple corpus. These corpora were each divided into training data and development data. As training data, the complex corpus has 181,095 sentences, and the simple corpus has 147,882 sentences. As development data, there were 22,637 sentences in the complex corpus and 18,485 sentences in the simple corpus.

5.1.2 Evaluation Data. For evaluation data, we need a parallel corpus to calculate the metrics described in 5.2. We used the WikiLarge corpus, the same as [43]. The WikiLarge corpus is a collection of [16] alignment sentence pairs, [46] alignment sentence pairs and revision sentences, and [34] WikiSmall. This parallel corpus is used as evaluation data by extracting only sentences that meet the above conditions. The evaluation data was a parallel corpus of 13,905 sentences.

\subsection{Evaluation}

We use three models with three evaluation metrics for comparison. The first is the Base model (3.1) and the second and third are the proposed model, specifically, Base+Pretrain model (base model plus the model in 3.2) and Base+Pretrain+Cycle model (base model plus the models in 3.2 and 3.3). For quantitative evaluation, we used three metrics: (a) FRE difference, (b) BLEU, and (c) SARI. These are explained below.

5.2.1 FRE difference. FRE [9] ${ }^{1}$ is a metric for evaluating the readability of a sentence based on the number of words and the number of syllables. The larger the value, the more readable the sentence.

\footnotetext{
${ }^{1}$ https://github.com/mmautner/readability
}

Table 1: Evaluation scores.

\begin{tabular}{|c|c|c|c|}
\hline & FRE difference & BLEU & SARI \\
\hline \hline Base & $\mathbf{+ 1 9 . 9 2}$ & 0.445 & 0.312 \\
\hline Base+Pretrain & +15.52 & 0.445 & 0.321 \\
\hline Base+Pretrain+Cycle & +9.98 & $\mathbf{0 . 4 5 3}$ & $\mathbf{0 . 3 2 2}$ \\
\hline
\end{tabular}

$F R E=206.835-1.015\left(\frac{\text { total } \text { words }}{\text { total sentences }}\right)-84.6\left(\frac{\text { total syllables }}{\text { total words }}\right)$

The FRE difference is an average value of FRE differences between input and output sentences. In other words, it can measure how simple it is compared to the input sentence.

5.2.2 BLEU. BLEU [24] is used to measure the meaning of the output and the correctness of its grammar by measuring the overlap between generated sentences and references. BLEU is defined by

$$
B L E U=e^{\left(1-\frac{r}{c}\right)} \exp \left(\sum_{n=1}^{N} w_{n} \log p_{n}\right),
$$

where $r$ is the reference sentence length, $c$ is the output sentence length, $w_{n}=1 / N$, and $p_{n}$ is the modified n-gram precisions. In the experiment, calculation is performed in 4-gram.

5.2.3 SARI. SARI [36 $]^{2}$ is a metric that measures the simplicity of output sentences. Evaluation of SARI requires a pair of three types of sentences, an input, a reference, and an output, and calculates the arithmetic mean of the precision and recall of the three rewrite operations (add, copy, delete) in n-gram.

\subsection{Model Parameters}

In all models, the embedding dimension is 100 , the style $s$ dimension is 200 , dimension of $z$ is 500 , and the dropout rate is 0.5 . The discriminator uses filters from 1 to 5 in size for the convolution layer, and has 128 filters each.

\section{RESULTS}

Table 1 shows the results of three models: FRE difference, BLEU, and SARI. As shown, the Base+Pretrain+Cycle model had the best performance in the evaluation with BLEU and SARI. By introducing pretrain reconstruction loss and cycle consistency loss, we can see that sentences with high grammaticality were generated closer to human sentence simplification. For the FRE difference, the Base model had the best performance. Next, we consider the FRE difference. Table 2 shows a part of the output of the actual model in order to qualitatively determine why the Base+Pretrain+Cycle model was inferior to the Base model.

Looking at the Base model output of Transfer Example 1 in Table 2, the value of FRE difference is large, as the number of words apparently decreased and simpler words were used, but the meaning of the whole sentence was not maintained. Furthermore, as price $\rightarrow$ process, the meaning at the word level could not be

\footnotetext{
${ }^{2}$ https://github.com/cocoxu/simplification
} 
Table 2: Example outputs from models.

\begin{tabular}{|c|c|c|}
\hline Transfer Example & Model & Sentence \\
\hline 1 & $\begin{array}{c}\text { Input } \\
\text { Reference } \\
\text { Base } \\
\text { Base+Pretrain } \\
\text { Base+Pretrain+Cycle }\end{array}$ & $\begin{array}{l}\text { the equilibrium price for a certain type of labor is the wage. } \\
\text { the balanced price for any kind of labor is called a wage. } \\
\text { the process for a source of one is the us wage. } \\
\text { the price of aerobic is a type of the labor wage. } \\
\text { the equilibrium factor for any type of the labor is wage. }\end{array}$ \\
\hline 2 & $\begin{array}{c}\text { Input } \\
\text { Reference } \\
\text { Base } \\
\text { Base+Pretrain } \\
\text { Base+Pretrain+Cycle }\end{array}$ & $\begin{array}{l}\text { he was diagnosed with inoperable abdominal cancer in april_num_. } \\
\text { he was diagnosed with abdominal cancer in april_num_. } \\
\text { he was diagnosed with donna brown in april_num_. } \\
\text { he was diagnosed with dead cancer in april_num_. } \\
\text { he was diagnosed with persistent cancer in april_num_. }\end{array}$ \\
\hline 3 & $\begin{array}{c}\text { Input } \\
\text { Reference } \\
\text { Base } \\
\text { Base+Pretrain } \\
\text { Base+Pretrain+Cycle }\end{array}$ & $\begin{array}{l}\text { finland rejected territorial demands and was invaded by the soviet union in november_num_. } \\
\text { finland rejected the soviets, so it was attacked in november_num_. } \\
\text { it was welcomed threat, and invaded the soviet union in november_num_. } \\
\text { finland did control and trade was fought by the soviet union in _num }{ }_{-} \text {. } \\
\text { finland was rejected libya and pushed by the soviet union in november_num_. }\end{array}$ \\
\hline
\end{tabular}

maintained. On the other hand, in the Base+Pretrain+Cycle model, the sentence length was long and conversion to simple words was less, but the retention of the sentence meaning and the correctness of the grammar were higher than that of the Base model.

In Transfer Example 2, the Base model performed a conversion (inoperable abdominal cancer $\rightarrow$ donna brown) whose meaning was not kept. However, in the Base+Pretrain+Cycle model, the conversion was inoerable $\rightarrow$ persistent, and thus the retention of the meaning was high.

Similarly, Transfer Example 3 was converted to finland rejected $\rightarrow$ it was welcomed in the Base model, and its meaning could not be maintained. On the other hand, in the Base+Pretrain+Cycle model, it was converted into invaded $\rightarrow$ pushed, and the retention of meaning was high.

In other words, each output of the Base model had a reduced number of words and many simple words. Although a high FRE difference was obtained, the FRE difference being inferior is not a big problem; the bigger problem is regarding grammaticality and the retention of meaning. From the above, we can see that the Base model had a reduced number of words and many simple words, but its retention of meaning and grammar was not preserved. In contrast, the proposed model (Base+Pretrain + Cycle) reduced the number of words within the range while retaining the meaning and grammar, and used simple words within it; thus, we can assume that the proposed model is better.

Also, in the output of development data in which there was no reference, there were a few transformations such as from "the faculty offers a broad range of academic programs and other training opportunities." to "the faculty offers a wide range of training and other training opportunities... Word substitution of the transfer (broad $\rightarrow$ wide, academic programmes $\rightarrow$ training) showed excellent results.

\section{CONCLUSION}

In this paper, we simplified sentences from a non-parallel corpus with adversarial learning in the framework of unsupervised style transfer in NLP. We collected sentences from simple-Wikipedia and en-Wikipedia articles and created a non-parallel corpus to create a model for sentence simplification. The proposed model
(Base+Pretrain+Cycle model), which introduces pretraining for reconstruction loss and cycle consistency loss, had a better performance in terms of BLEU and SARI than the base model. Moreover, the comparison in the output sentences showed more effective simplification and content retention in our model than in the base model. In the future, we plan to modify the generator's model structure for problem complication (e.g., to remove the word limit).

\section{ACKNOWLEDGMENTS}

We gratefully acknowledge Associate Professor Mamoru Komachi for their helpful comments and suggestions.

\section{REFERENCES}

[1] Mikel Artetxe, Gorka Labaka, Eneko Agirre, and Kyunghyun Cho. 2018. Unsupervised Neural Machine Translation. In International Conference on Learning Representations. https://openreview.net/forum?id=Sy2ogebAW

[2] Or Biran, Samuel Brody, and Noemie Elhadad. 2011. Putting it Simply: a ContextAware Approach to Lexical Simplification. In Proceedings of the 49th Annual Meeting of the Association for Computational Linguistics: Human Language Technologies. Association for Computational Linguistics, Portland, Oregon, USA, 496-501. https://www.aclweb.org/anthology/P11-2087

[3] Arnaldo Candido, Jr., Erick Maziero, Caroline Gasperin, Thiago A. S. Pardo, Lucia Specia, and Sandra M. Aluisio. 2009. Supporting the Adaptation of Texts for Poor Literacy Readers: A Text Simplification Editor for Brazilian Portuguese. In Proceedings of the Fourth Workshop on Innovative Use of NLP for Building Educational Applications (EdAppsNLP'09). Association for Computational Linguistics, Stroudsburg, PA, USA, 34-42. http://dl.acm.org/citation.cfm?id=1609843.1609848

[4] John Carroll, Guido Minnen, Darren Pearce, Yvonne Canning, Siobhan Devlin, and John Tait. 1999. Simplifying Text for Language-Impaired Readers. In In Proceedings of the 9th Conference of the European Chapter of the Association for Computational Linguistics (EACL. 269-270.

[5] R. Chandrasekar, Christine Doran, and B. Srinivas. 1996. Motivations and Methods for Text Simplification. In Proceedings of the 16th Conference on Computational Linguistics - Volume 2 (COLING '96). Association for Computational Linguistics, Stroudsburg, PA, USA, 1041-1044. https://doi.org/10.3115/993268.993361

[6] Yong Cheng, Zhaopeng Tu, Fandong Meng, Junjie Zhai, and Yang Liu. 2018. Towards Robust Neural Machine Translation. In Proceedings of the 56th Annual Meeting of the Association for Computational Linguistics (Volume 1: Long Papers). Association for Computational Linguistics, Melbourne, Australia, 1756-1766. https://www.aclweb.org/anthology/P18-1163

[7] William Fedus, Ian Goodfellow, and Andrew M. Dai. 2018. MaskGAN: Better Text Generation via Filling in the ___. In International Conference on Learning Representations. https://openreview.net/forum?id=ByOExmWAb

[8] Katja Filippova and Michael Strube. 2008. Dependency Tree Based Sentence Compression. In Proceedings of the Fifth International Natural Language Generation Conference. Association for Computational Linguistics, Salt Fork, Ohio, USA, 25-32. https://www.aclweb.org/anthology/W08-1105 
[9] Rudolph Flesch. 1948. A new readability yardstick. In Journal of applied psychology. 32(3):221.

[10] Goran Glavaš and Sanja Štajner. 2015. Simplifying Lexical Simplification: Do We Need Simplified Corpora?. In Proceedings of the 53rd Annual Meeting of the Association for Computational Linguistics and the 7th International Joint Conference on Natural Language Processing (Volume 2: Short Papers). Association for Computational Linguistics, Beijing, China, 63-68. https://doi.org/10.3115/v1/P15-2011

[11] Ian Goodfellow, Jean Pouget-Abadie, Mehdi Mirza, Bing Xu, David Warde-Farley, Sherjil Ozair, Aaron Courville, and Yoshua Bengio. 2014. Generative Adversarial Nets. In Advances in Neural Information Processing Systems 27, Z. Ghahramani, M. Welling, C. Cortes, N. D. Lawrence, and K. Q. Weinberger (Eds.). Curran Associates, Inc., 2672-2680. http://papers.nips.cc/paper/5423-generative-adversarialnets.pdf

[12] Zhiting Hu, Zichao Yang, Xiaodan Liang, Ruslan Salakhutdinov, and Eric P. Xing. 2017. Toward Controlled Generation of Text. In Proceedings of the 34th International Conference on Machine Learning (Proceedings of Machine Learning Research), Doina Precup and Yee Whye Teh (Eds.), Vol. 70. PMLR, International Convention Centre, Sydney, Australia, 1587-1596. http://proceedings.mlr.press/ v70/hu17e.html

[13] Kentaro Inui, Atsushi Fujita, Tetsuro Takahashi, Ryu Iida, and Tomoya Iwakura 2003. Text Simplification for Reading Assistance: A Project Note. In Proceedings of the Second International Workshop on Paraphrasing - Volume 16 (PARAPHRASE '03). Association for Computational Linguistics, Stroudsburg, PA, USA, 9-16. https://doi.org/10.3115/1118984.1118986

[14] Harsh Jhamtani, Varun Gangal, Eduard Hovy, and Eric Nyberg. 2017. Shakespearizing Modern Language Using Copy-Enriched Sequence to Sequence Models. In Proceedings of the Workshop on Stylistic Variation. Association for Computational Linguistics, Copenhagen, Denmark, 10-19. https://doi.org/10.18653/v1/ W17-4902

[15] Nobuhiro Kaji, Daisuke Kawahara, Sadao Kurohash, and Satoshi Sato. 2002. Verb Paraphrase Based on Case Frame Alignment. In Proceedings of the 40th Annual Meeting on Association for Computational Linguistics (ACL '02). Association for Computational Linguistics, Stroudsburg, PA, USA, 215-222. https://doi.org/10. 3115/1073083.1073120

[16] David Kauchak. 2013. Improving Text Simplification Language Modeling Using Unsimplified Text Data. In Proceedings of the 51st Annual Meeting of the Association for Computational Linguistics (Volume 1: Long Papers). Association for Computational Linguistics, Sofia, Bulgaria, 1537-1546. https://www.aclweb.org/ anthology/P13-1151

[17] Beata Beigman Klebanov, Kevin Knight, and Daniel Marcu. 2004. Text Simplification for Information-Seeking Applications. In On the Move to Meaningful Internet Systems 2004: CoopIS, DOA, and ODBASE, OTM Confederated Interna tional Conferences, Agia Napa, Cyprus, October 25-29, 2004, Proceedings, Part I. 735-747. https://doi.org/10.1007/978-3-540-30468-5 47

[18] Juncen Li, Robin Jia, He He, and Percy Liang. 2018. Delete, Retrieve, Generate: a Simple Approach to Sentiment and Style Transfer. In Proceedings of the 2018 Conference of the North American Chapter of the Association for Computational Linguistics: Human Language Technologies, Volume 1 (Long Papers). Association for Computational Linguistics, New Orleans, Louisiana, 1865-1874. https://doi org/10.18653/v1/N18-1169

[19] Jiwei Li, Will Monroe, Tianlin Shi, Sébastien Jean, Alan Ritter, and Dan Jurafsky. 2017. Adversarial Learning for Neural Dialogue Generation. In Proceedings of the 2017 Conference on Empirical Methods in Natural Language Processing. Association for Computational Linguistics, Copenhagen, Denmark, 2157-2169. https://doi.org/10.18653/v1/D17-1230

[20] Ming-Yu Liu and Oncel Tuzel. 2016. Coupled Generative Adversarial Networks. In Advances in Neural Information Processing Systems 29, D. D. Lee, M. Sugiyama, U. V. Luxburg, I. Guyon, and R. Garnett (Eds.). Curran Associates, Inc., 469-477. http://papers.nips.cc/paper/6544-coupled-generative-adversarial-networks.pdf

[21] Tomas Mikolov, Ilya Sutskever, Kai Chen, Greg S Corrado, and Jeff Dean. 2013 Distributed Representations of Words and Phrases and their Compositionality. In Advances in Neural Information Processing Systems 26, C. J. C. Burges, L. Bottou, M. Welling, Z. Ghahramani, and K. Q. Weinberger (Eds.). Curran Associates, Inc., 3111-3119.

[22] Shashi Narayan and Claire Gardent. 2014. Hybrid Simplification using Deep Semantics and Machine Translation. In Proceedings of the 52nd Annual Meeting of the Association for Computational Linguistics (Volume 1: Long Papers). Association for Computational Linguistics, Baltimore, Maryland, 435-445. https://doi.org/10 3115/v1/P14-1041

[23] Gustavo H. Paetzold and Lucia Specia. 2016. Unsupervised Lexical Simplification for Non-native Speakers. In Proceedings of the Thirtieth AAAI Conference on Artificial Intelligence (AAAI'16). AAAI Press, 3761-3767. http://dl.acm.org/ citation.cfm?id=3016387.3016433

[24] Kishore Papineni, Salim Roukos, Todd Ward, and Wei jing Zhu. 2002. BLEU: a Method for Automatic Evaluation of Machine Translation. 311-318.

[25] Shrimai Prabhumoye, Yulia Tsvetkov, Ruslan Salakhutdinov, and Alan W. Black. 2018. Style Transfer Through Back-Translation. In Proceedings of the 56th Annual
Meeting of the Association for Computational Linguistics (Volume 1: Long Papers). Association for Computational Linguistics, Melbourne, Australia, 866-876. https: //www.aclweb.org/anthology/P18-1080

[26] Bangalore Srinivas Raman Chandrasekar. [n. d.]. Automatic induction of rules for text simplification. Knowledge-Based Systems, 10(3), 183-190.

[27] Scott Reed, Zeynep Akata, Xinchen Yan, Lajanugen Logeswaran, Bernt Schiele, and Honglak Lee. 2016. Generative Adversarial Text to Image Synthesis. In Proceedings of The 33rd International Conference on Machine Learning (Proceedings of Machine Learning Research), Maria Florina Balcan and Kilian Q. Weinberger (Eds.), Vol. 48. PMLR, New York, New York, USA, 1060-1069. http://proceedings. mlr.press/v48/reed16.html

[28] Alexander M. Rush, Sumit Chopra, and Jason Weston. 2015. A Neural Attention Model for Abstractive Sentence Summarization. In Proceedings of the 2015 Conference on Empirical Methods in Natural Language Processing. Association for Computational Linguistics, Lisbon, Portugal, 379-389. https://doi.org/10.18653/v1/D151044

[29] Sergiu Nisioi Sanja tajner. 2018. A Detailed Evaluation of Neural Sequence-toSequence Models for In-domain and Cross-domain Text Simplification. In In Proceedings of the Eleventh International Conference on Language Resources and Evaluation. LREC, Miyazaki, Japan.

[30] R. Barzilay T. Shen, T. Lei and T. Jaakkola. 2017. Style transfer from non-parallel text by cross-alignment. In Proceedings of the The 8th International foint Conference on Natural Language Processing. ACL, 6833-6844.

[31] Hong Yu Tsendsuren Munkhdalai. 2017. Neural semantic encoders. In In Proceedings of the 15th Conference of the European Chapter of the Association for Computational Linguistics. Association for Computational Linguistics, 397-407.

[32] Tu Vu, Baotian Hu, Tsendsuren Munkhdalai, and Hong Yu. 2018. Sentence Simplification with Memory-Augmented Neural Networks. In Proceedings of the 2018 Conference of the North American Chapter of the Association for Computational Linguistics: Human Language Technologies, Volume 2 (Short Papers). Association for Computational Linguistics, New Orleans, Louisiana, 79-85. https://doi.org/10.18653/v1/N18-2013

[33] Tong Wang, Ping Chen, John Rochford, and Jipeng Qiang. 2016. Text Simplification Using Neural Machine Translation. In Proceedings of the Thirtieth AAAI Conference on Artificial Intelligence (AAAI'16). AAAI Press, 4270-7271. http://dl.acm.org/citation.cfm?id=3016387.3016551

[34] Kristian Woodsend and Mirella Lapata. 2011. Learning to Simplify Sentences with Quasi-synchronous Grammar and Integer Programming. In Proceedings of the Conference on Empirical Methods in Natural Language Processing (EMNLP '11). Association for Computational Linguistics, Stroudsburg, PA, USA, 409-420. http://dl.acm.org/citation.cfm?id=2145432.2145480

[35] Sander Wubben, Antal van den Bosch, and Emiel Krahmer. 2012. Sentence Simplification by Monolingual Machine Translation. In Proceedings of the 50th Annual Meeting of the Association for Computational Linguistics: Long Papers Volume 1 ( $A C L$ '12). Association for Computational Linguistics, Stroudsburg, PA, USA, 1015-1024. http://dl.acm.org/citation.cfm?id=2390524.2390660

[36] Wei Xu, Courtney Napoles, Ellie Pavlick, Quanze Chen, and Chris CallisonBurch. 2016. Optimizing Statistical Machine Translation for Text Simplification. Transactions of the Association for Computational Linguistics 4 (Dec. 2016), 401415. https://doi.org/10.1162/tacl_a_00107

[37] Zhen Xu, Bingquan Liu, Baoxun Wang, Chengjie SUN, Xiaolong Wang, Zhuoran Wang, and Chao Qi. 2017. Neural Response Generation via GAN with an Approximate Embedding Layer. In Proceedings of the 2017 Conference on Empirical Methods in Natural Language Processing. Association for Computational Linguistics, Copenhagen, Denmark, 617-626. https://doi.org/10.18653/v1/D17-1065

[38] Zhen Yang, Wei Chen, Feng Wang, and Bo Xu. 2018. Improving Neural Machine Translation with Conditional Sequence Generative Adversarial Nets. In Proceedings of the 2018 Conference of the North American Chapter of the Association for Computational Linguistics: Human Language Technologies, Volume 1 (Long Papers). Association for Computational Linguistics, New Orleans, Louisiana, 1346-1355. https://doi.org/10.18653/v1/N18-1122

[39] Zichao Yang, Zhiting Hu, Chris Dyer, Eric P Xing, and Taylor Berg-Kirkpatrick. 2018. Unsupervised Text Style Transfer using Language Models as Discriminators. In Advances in Neural Information Processing Systems 31, S. Bengio, H. Wallach, H. Larochelle, K. Grauman, N. Cesa-Bianchi, and R. Garnett (Eds.). Curran Associates, Inc., 7287-7298.

[40] Mark Yatskar, Bo Pang, Cristian Danescu-Niculescu-Mizil, and Lillian Lee. 2010. For the sake of simplicity: Unsupervised extraction of lexical simplifications from Wikipedia. In Human Language Technologies: The 2010 Annual Conference of the North American Chapter of the Association for Computational Linguistics. Association for Computational Linguistics, Los Angeles, California, 365-368. https://www.aclweb.org/anthology/N10-1056

[41] Lantao Yu, Weinan Zhang, Jun Wang, and Yong Yu. 2016. SeqGAN: Sequence Generative Adversarial Nets with Policy Gradient. CoRR abs/1609.05473 (2016). http://dblp.uni-trier.de/db/journals/corr/corr1609.html\#YuZWY16

[42] John Tait Yvonne Canning. [n. d.]. Syntactic simplification of newspaper text for aphasic readers. In Customised Information Delivery, 6-11. 
[43] Xingxing Zhang and Mirella Lapata. 2017. Sentence Simplification with Deep Reinforcement Learning. In Proceedings of the 2017 Conference on Empirical Methods in Natural Language Processing. Association for Computational Linguistics, Copenhagen, Denmark, 584-594. https://doi.org/10.18653/v1/D17-1062

[44] Yizhe Zhang, Zhe Gan, Kai Fan, Zhi Chen, Ricardo Henao, Dinghan Shen, and Lawrence Carin. 2017. Adversarial Feature Matching for Text Generation. In Proceedings of the 34th International Conference on Machine Learning (Proceeding of Machine Learning Research), Doina Precup and Yee Whye Teh (Eds.), Vol. 70 PMLR, International Convention Centre, Sydney, Australia, 4006-4015. http: //proceedings.mlr.press/v70/zhang17b.html

[45] Park T. Isola P. Zhu, J.-Y. and A. A. Efros. 2017. Unpaired Image-to-Image Translation using Cycle-Consistent Adversarial Networks. ICCV.

[46] Zhemin Zhu, Delphine Bernhard, and Iryna Gurevych. 2010. A Monolingual Tree-based Translation Model for Sentence Simplification. In Proceedings of the 23rd International Conference on Computational Linguistics (COLING '10). Association for Computational Linguistics, Stroudsburg, PA, USA, 1353-1361. http://dl.acm.org/citation.cfm?id=1873781.1873933 